\title{
Image Processing
}

National Cancer Institute

\section{Source}

National Cancer Institute. Image Processing. NCI Thesaurus. Code C17606.

The use of data processing systems to analyze, enhance, interpret or display digital images. 